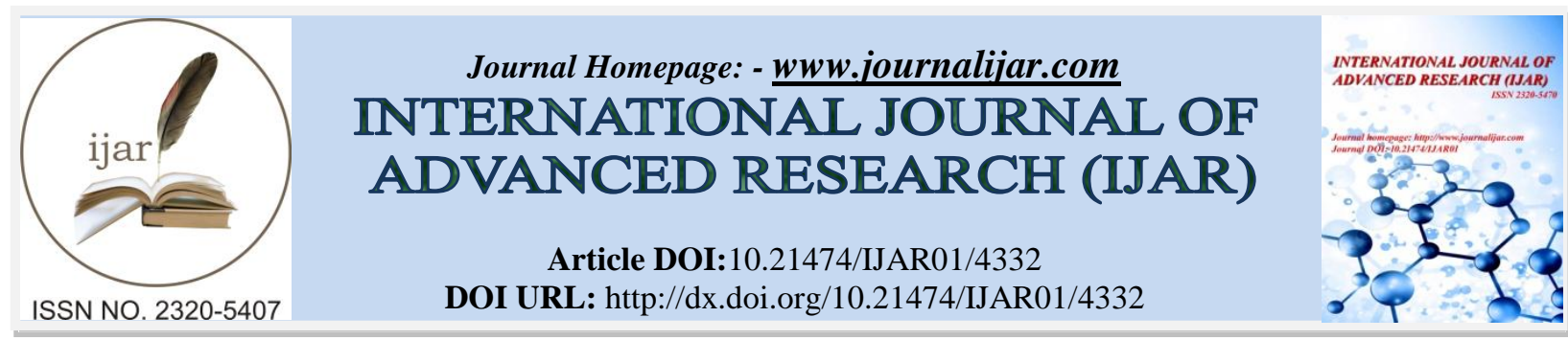

RESEARCH ARTICLE

\title{
SEDIMENTOLOGIE DE FACIES DES DEPOTS TRIASIQUES DE LA REGION DE OUED EL MALEH ET ELGARA (BASSIN DE MOHAMMEDIA-BENSLIMANE-ELGARA-BERRECHID, MAROC).
}

\author{
Abdelkrim Afenzar and Rachid Essamoud. \\ Laboratoire de Dynamique des bassins sédimentaires et corrélations géologiques, Faculté des Sciences Ben M'sik, \\ Université Hassan II de Casablanca. B.P. 7955, Sidi Othmane, Casablanca, Maroc.
}

\section{Manuscript Info}

..........................

ManuscriptHistory

Received: 26 March 2017

Final Accepted: 31 April 2017

Published: May 2017

Key words:-

Région de Oued El Maleh-ElGara,

Meseta marocaine, Trias, analyse

sédimentologique, élément architectural,

paléoenvironnement.

\section{Abstract}

The Triassic deposits of the Mohammedia-Benslimane-ElGaraBerrechidbasin have a silicoclastic and saliferous nature covered by basaltic flows. These deposits occur locally in the northern, eastern and southern parts of the basin, and plunge weakly towards the south west under cretaceous deposits. In this paper, we are interested in the Triassic outcrops at Oued El Maleh-ElGara area (central and northern part of the basin). The detailed description of the outcrops and the recognition of facies and architectural elements characteristic of the deposition environment revealed the existence of a clastic and evaporitic episode before the basalt effusion.

This study has shown an important palaeoenvironmental evolution that has evolved from an alluvial fan environment (the base of the series) to a braided river environment that becomes anastomosed (middle of the series). At the end, the system moved to a coastal plain environment characterized by very fine and evaporitic deposits resulting from an installation of lacustrine and also lagoonal environments in connection with a Upper-Triassic epicontinental sea.

Copy Right, IJAR, 2017,. All rights reserved.

\section{Introduction:-}

Les dépôts détritiques terrigènes et évaporitique du Trias du Maroc présentent des enjeux scientifiques et économiques considérables.

La série triasique dans cette région d'étude est formée essentiellement par des dépôts détritiques, argileux et évaporitiques rarement fossilifères (Lyazidi, 2004). La description, la classification et l'interprétation des dépôts sont basées sur plusieurs critères: hiérarchies de strates et de leurs surfaces de délimitation, lithofaciès, la géométrie des corps sédimentaires, les associations de lithofaciès (éléments architecturaux) (Miall, 1985; Bridge, 1993; Miall 2006). L'ensemble de ces caractéristiques permettent la reconstitution des paléoenvironnements.

Une analyse sédimentologique de faciès constitue la base de toute analyse de bassin. Malgré l'importance de cette démarche ainsi que la richesse de cette région en terme de substances utiles (sel gemme, sel potassique, argiles, ...etc.), aucune étude sédimentologique bien détaillée n'a été faite dans la zone d'étude. Ceci est la raison qui nous a amené à entamer cette étude, ce qui va nous permettre de faire en plus des corrélations avec d'autres bassins triasiques à l'échelle régionale et même globale.

Corresponding Author:-Abdelkrim Afenzar.

Address:-Laboratoire de Dynamique des bassins sédimentaires et corrélations géologiques, Faculté des Sciences Ben M’sik, Université Hassan II de Casablanca. B.P. 7955, Sidi Othmane, Casablanca, Maroc. 


\section{Situation géographique et cadre géologique:-}

La région de Oued El Maleh-El Gara, se situe au nord-ouest de la Meseta côtière marocaine, à une vingtaine de kilomètres au nord-est de Casablanca et une trentaine de kilomètres au sud de Benslimane. Cette région fait partie du bassin triasique de Mohammedia-Benslimane-ElGara-Berrechid qui se présente comme une vaste dépression peu profonde qui semble relever d'une structure en demi-grabens de direction N-S à NE-SW, dont la disposition géographique épouse la direction des structures majeures hercyniennes (El Wartiti et al.,1992; Lyazidi, 2004).

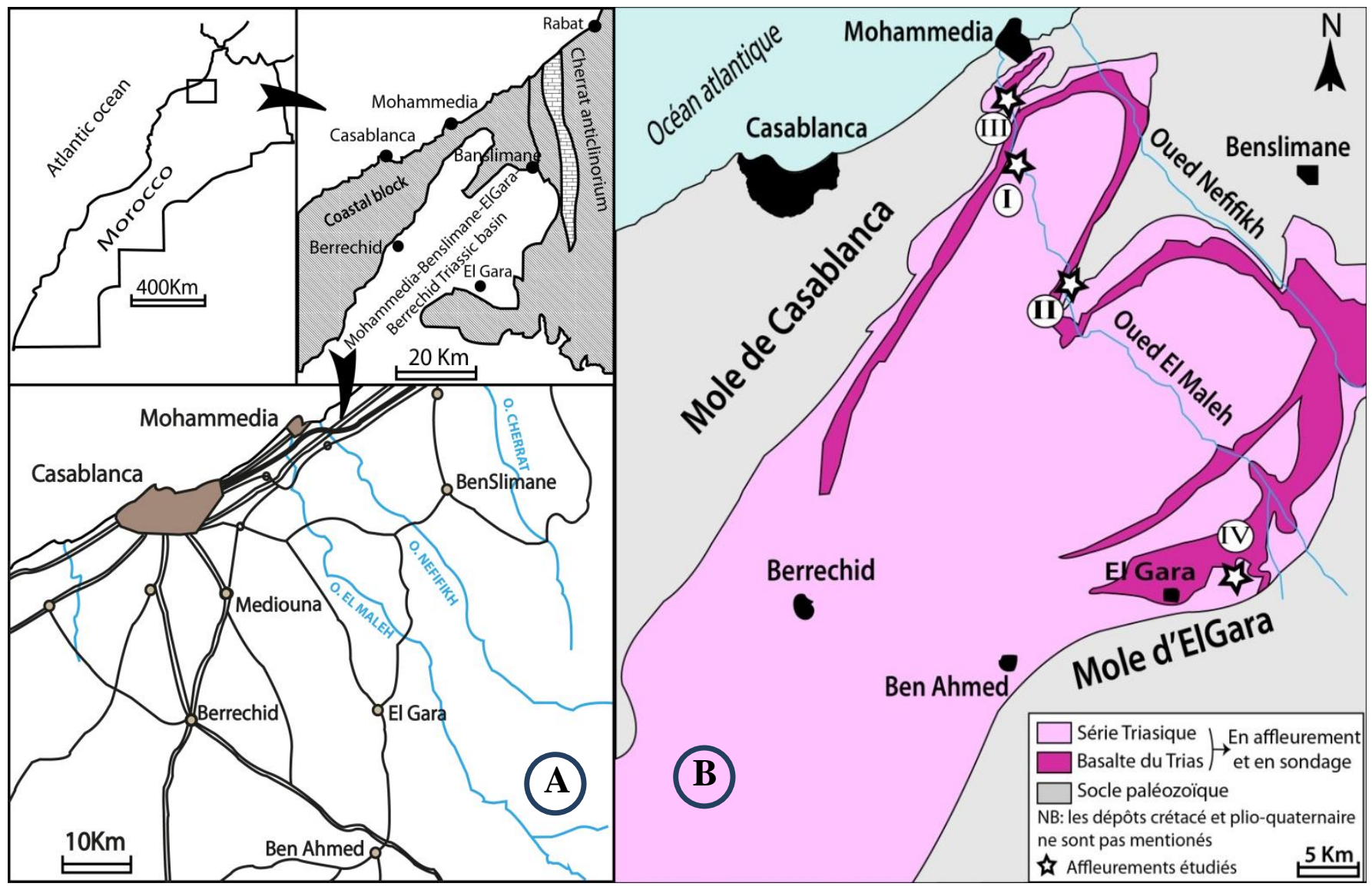

Figure 1:- Localisation géographique (carte A) et situation géologique de la zone d'étude dans la carte d'extention du bassin triasique sous la couverture (carte B : Lyazidi, 2004. modifiée) .

La zone d'étude est limitée à l'ouest, par le socle cambro-ordovicien du môle côtier (Destombes et Jeannette, 1966) sur lequel est déposée la série triasique en discordance, à l'est par les collines des Khatouat (Fadli, 1990), au nord par la zone structurée de Benslimane (Piqué, 1979 ; Zahraoui, 1991), et au nord-est par la zone de Oued Cherrat (Piqué, 1979 ; Chalouan, 1981 ; Zahraoui, 1991).

Selon Manspeizer (1988), cette zone d'étude qui appartient à la meseta occidentale marocaine, est étroitement liée à la marge atlantique ouest marocaine. La majorité des dépôts dans cette zone, sont d'origine détritique terrigène et évaporitique, déposés au Trias qui est considéré comme une période importante dans l'histoire géologique du Maroc, en raison des phénomènes qui s'y sont déroulés en relation avec l'ouverture de l'atlantique centrale (Medina, 1996).

\section{Méthodologie:-}

Cette étude consiste en une analyse fine des coupes de terrain pour une meilleure vision des corps sédimentaires et leurs agencements dans l'espace afin de caractériser les différents faciès et leurs associations ainsi que les éléments architecturaux (Miall, 1985, 2006) qui nous permettront de reconstituer les environnements de dépôt de façon précise. Dans cette étape nous nous sommes basés sur les caractères lithologiques, granulométriques, les textures et 
les structures sédimentaires observés à l'affleurement. La nomenclature des faciès dans cette étude est comparée avec celle établie par Miall (1977, 1978 et 2006).

L'agencement vertical et horizontal de ces faciès donne naissance à ce qu'on appelle les éléments architecturaux. Ces derniers sont caractérisés selon plusieurs critères déterminés par Allen, (1983) et Miall, (1985, 2006), et qui sont: la nature des surfaces de délimitation inférieure et supérieure, la géométrie externe, l'échelle et finalement la géométrie interne. La dernière étape consiste à la détermination des paléoenvironnements en se basant sur la nature et les types des lithofaciès déjà caractérisés et les types des éléments architecturaux formés par ces lithofaciès.

\section{Résultats de l'analyse faciologique:-}

\section{Lithofaciès:-}

Faciès grossiers (conglomérat):-

Faciès F1: Ce faciès affleure à la base de la coupe de Sidi Bouchaibe dans la rive droite de Oued El Maleh. Il s'agit d'un conglomérat violacé, massif, à éléments anguleux de taille de 2 à $15 \mathrm{~cm}$, non organisés et mal triés, ceci est probablement lié à une forte viscosité qui inhibe la turbulence et les interactions entre les grains et à cause d'un mécanisme de transport assez court, l'ensemble flotte dans une matrice formée essentiellement de grès très fins. Ce faciès correspond au faciès Gms de Miall (1978, 1985, 2006). Selon Miall (1977, 1978); Poli (1997); Opluštil $\boldsymbol{e t}$ al. (2005); Miall (2006), ce type de conglomérat peut se déposer par des écoulements gravitaires sous forme de coulés de débris (debris flow) au niveau des cônes alluviaux. D'après Sohn (2000), ce type de coulés de débris été interprété selon deux modèles, celui d'écoulement visco-plastique de Johnson (1984) et/ou d'écoulement interstitiel des grains de Takahashi $(\mathbf{1 9 7 8 , 1 9 8 1 , 1 9 9 1 )}$.

Tableau 1:- Description et interprétation des faciès sédimentaires (d'après Miall, 1985, 2006, modifié).

\begin{tabular}{|l|l|l|}
\hline Lithofaciès & Caractéristiques & Interpretation \\
\hline FacièsConglomératiques & \\
\hline F1 : Gms & Conglomérat massif mal organisé, mal trié & $\begin{array}{l}\text { Ecoulements de debris, dépôts } \\
\text { d'écoulement gravitaires }\end{array}$ \\
\hline F2a : Gm & Conglomérat stratifié, imbrication des galets & Dépôts de barreslongitudinales \\
\hline F2b : Gm & Conglomérats organisés pauvre en matrice & $\begin{array}{l}\text { Dépôts tamisés, dépôts du fond de } \\
\text { chenal }\end{array}$ \\
\hline Facièsgréseux & Dépôtsd'écoulementgravitaires \\
\hline F3 : Sm & Grès massif, fine à grossier & Régime d'écoulementsupérieur \\
\hline F4 : Sh & $\begin{array}{l}\text { Grès à litages plans horizontaux, } \\
\text { très fine à moyen }\end{array}$ & Dépôtsd'overbank \\
\hline Facièssilto-argileux & $\begin{array}{l}\text { Dépôts d'overbank ou de chenaux } \\
\text { abandonnés }\end{array}$ \\
\hline F5 : Fl & Siltites laminées à massives & \\
\hline F6 : Fm & Argilitesmassivesoulaminées & Dépôts lagunaires ou de Playa lake \\
\hline Faciès évaporitique & \\
\hline F7 & Gypse fibreux (Intercalé dans les argilites) \\
\hline
\end{tabular}

Faciès $\boldsymbol{F 2 a}$ : Le faciès $\boldsymbol{F 2 a}$ a été identifié à la base des affleurements étudié (coupes I, II et III, fig. 2) avec une épaisseur variable entre 0,5 et $2 \mathrm{~m}$. C'est un lithofaciès conglomératique violacé à éléments centimétriques subanguleux (galets et graviers de nature quartzitique et gréseuse). Les galets et les graviers sont stratifiés et parfois imbriqués dans une matrice gréseuse très peu abondante. Ces caractéristiques montrent que ce conglomérat correspond au faciès $\mathbf{G m}$ de Miall $(\mathbf{1 9 7 8 , ~ 1 9 8 5 , ~ 2 0 0 6 ) . ~ C e ~ t y p e ~ d e ~ f a c i e ̀ s ~ s e ~ d e ́ p o s e ~ a u ~ n i v e a u ~ d e s ~ b a r r e s ~}$ longitudinales dans un système fluviatile en tresse (Rust, 1972, 1978, 1981; Miall 1977, 1978, 2006; Reineck et Singh, 1980).

Faciès $\boldsymbol{F 2} \boldsymbol{b}$ : Ce faciès $(0,5$ à $2 \mathrm{~m})$ conglomératique violacé montre une abondance des galets avec une quasi absence de la matrice, ils sont complètement vannés et vraisemblablement déposés en nappe. Les caractéristiques de ce faciès ressemblent à celle déterminées par Miall $(1977,1978,2006)$ pour le faciès $\mathbf{G m}$ et peuvent correspondre à des dépôts du fond tamisés "sieve deposits". 
Faciès moyens (faciès gréseux):-

Faciès F3: Le faciès F3 a été identifié dans la coupe de Kachlat Arian au niveau du barrage de Oued El Maleh. C'est un grès grossier massif, de 2 à $3 \mathrm{~m}$ d'épaisseur. Il présente parfois des fragments millimétriques à centimétriques à la base où il tend vers un microconglomérat. Ce faciès est caractérisé principalement par son aspect massif et par l'absence de structures sédimentaires. La présence de fragments surdimensionnés isolés est probablement liée à leur chute le long de la pente (Sohn $\boldsymbol{e t ~ a l . , ~ 1 9 9 7 ) ~ a s s o c i e ́ e ~ a u ~ m e ́ c a n i s m e ~ d e ~ d e ́ p o ̂ t ~ l u i - m e ̂ m e ~ o u ~}$ au mouvement dans un écoulement de forte charge (Postma et Cruickshank, 1988). Ce faciès correspond au faciès Sm de Miall (1978, 1985, 2006) et Einsele (2000). D'après Einsele, (2000); Eji Uba et al. (2005); Miall (2006); Menkemet al $\mathbf{( 2 0 1 3 )}$ ce faciès correspond à un dépôt rapide en période de fortes inondations ou un dépôt par des écoulements gravitaires.

Faciès F4: Ce faciès a été identifié dans tous les affleurements du bassin. C'est un grès violacé, moyen à très fin en alternance souvent avec des faciès argileux. Il est caractérisé par une épaisseur moyenne $(10 \mathrm{à} 60 \mathrm{~cm})$, et par des laminations horizontales parallèles avec des linéations de délit (Partinglineation). Cette structure est générée par de petits tourbillons longitudinaux à la base de la couche turbulente interne, et fournit un excellent indicateur de paléocourant (Miall, 2006). Selon Miall $(\mathbf{1 9 7 8 , 1 9 9 6 )}$ ce faciès a été interprété comme étant le résultat d'un dépôt par traction en régime d'écoulement supérieur. Par ceci le faciès F4 ressemble au faciès Sh de Miall (1977, 1978, 2006).

Faciès fins:-

Faciès F5: Ce faciès est représenté par des siltites et siltites gréseuses à lamination horizontale en alternance parfois avec des minces niveaux du grès fin. Dans d'autres cas, ces siltites sont massives avec une épaisseur de 1 à $5 \mathrm{~m}$. Elles ont une couleur rougeâtre avec des niveaux de marmorisation verdâtres à grisâtres dues à la transformation du fer dans le sédiment. Ce faciès atteint son épaisseur maximale dans la région d'ElGara (coupe d'Oulad Jhaych) et dans la région de l'Oued El Maleh (coupe de Kachlat Arian). Le faciès F5 présente une grande ressemblance avec le faciès Fl de Miall (1978; 2006). Ce faciès correspond à un dépôt d'accrétion verticale caractéristique d'un écoulement laminaire de très faible énergie (Miall, 1977; 1978; 2006). On peut donc interpréter ce faciès comme un dépôt de plaine alluviale aride ou de chenaux abandonnés.

\section{Faciès F6:-}

Il s'agit des argilites massives rougeâtres montrant des tâches de marmorisation avec l'absence de stratification et lamination. Ce faciès a été identifié dans tous les affleurements étudiés dans le bassin avec un changement d'épaisseur d'une région à l'autre. Dans la région du barrage de l'Oued El Maleh, ce faciès atteint son épaisseur maximale qui est de l'ordre de la vingtaine de mettre, alors que son épaisseur minimale est de l'ordre de $40 \mathrm{~cm}$. Ces argilites contiennent parfois des remplissages de fractures par du gypse (coupe d'OuledJhaich dans la région d'ElGara). Ce faciès qui correspond au faciès $\mathbf{F m}$ a été interprété comme un dépôt d'overbank dans une plaine d'inondation (Miall, 1978, 1985, 2006; Einsele, 2000 ; Eji Uba et al., 2005).

\section{Faciès Evaporitiques:-}

Faciès F7: Il s'agit d'un lithofaciès de gypse fibreux centimétrique qui alterne dans la majorité des cas avec les faciès F5: Fl et F6: Fm. Il est identifié au niveau de la partie médiane et sommitale de la série triasique dans le bassin. Il est généralement formé dans des environnements relativement arides. Ce faciès est formé par la précipitation d'ions en solution, dans un milieu sursaturé (saumure) soumis à une évaporation intense (Warren, $2006 ; 2010$ ). 

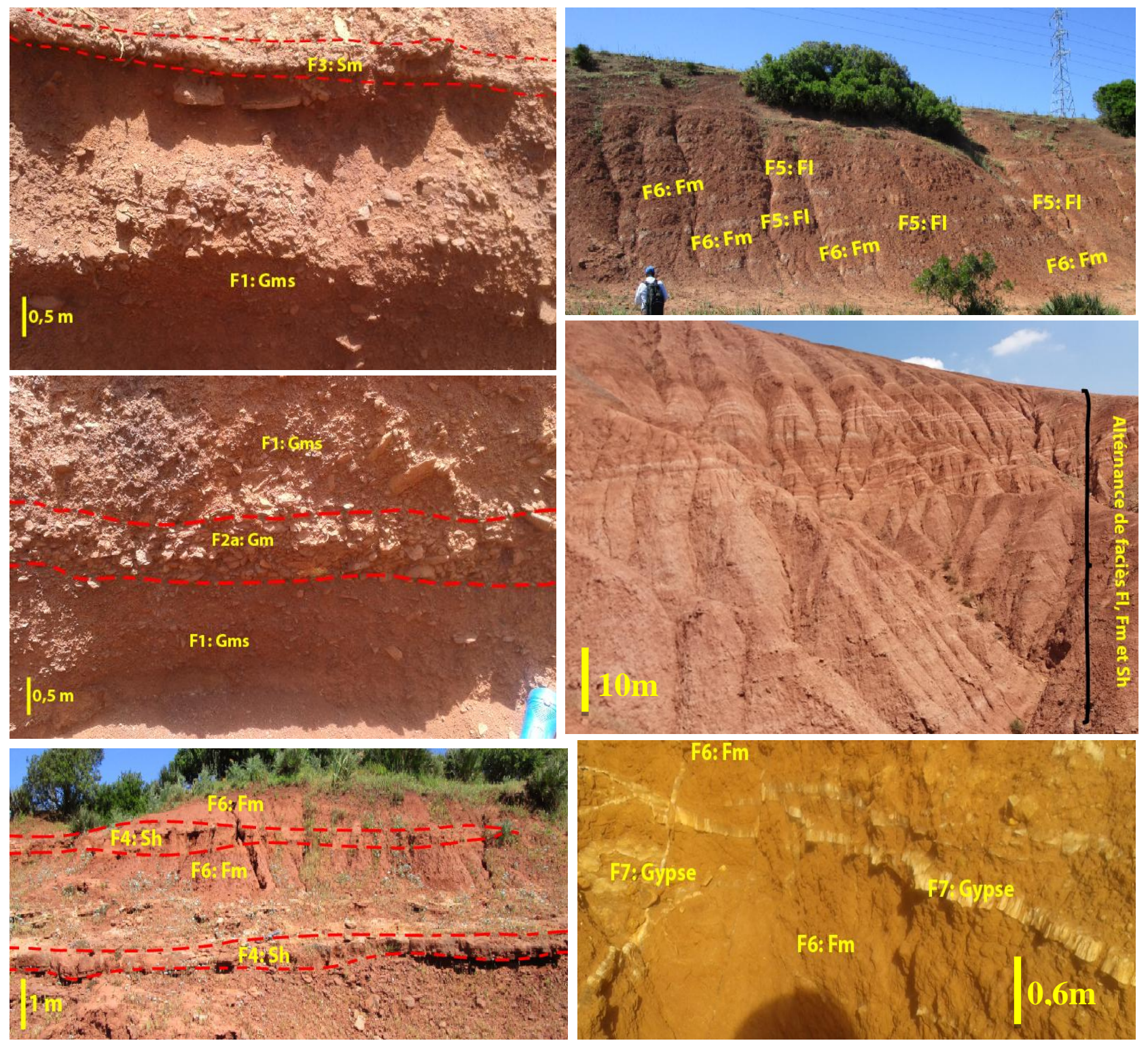

Figure 2:- Photos des faciès identifiés dans la région de l'oued Maleh-ElGara.

Eléments architecturaux:-

Elément architectural SG "Sediment-Gravity-Flow":-

Cet élément est caractérisé à la base de la série, au niveau de l'affleurement de Sidi Bouchaibe dans la région de Oued El Maleh. Il est formé principalement par l'association des faciès F1: Gms et F2a: Gm. Selon Miall (1985, 2006), il est formé par des dépôts d'écoulement gravitaire, principalement des galets et des graviers mal triés supporté par une matrice abondante de nature gréseuse. Cet élément a été déposé dans les zones proximales des cônes de déjection.

\section{Elément architectural GB "Gravel Bars and Bedforms":-}

L'élément architectural GB été identifié à la base de la coupe de Kachlat Arian et de Sidi Bou Amar. Il est formé par l'association des lithofaciès grossiers qui contiennent des galets et des graviers imbriqués et stratifiés (lithofaciès F2a:Gm). Parfois des lithofaciès mineurs ont été identifié entre ces faciès majeurs (exemple de lithofaciès F3: Sm et F4: Sh). La majorité des faciès de cet élément sont organisés en corps tabulaires de cinq à six mètres d'épaisseur. Il est formé également d'un pourcentage de 4 à $6 \%$ de grès fins à moyens. Il s'agit donc de dépôts grossiers déposés 
au niveau des barres (barres graveleuses), et qui sont parfois intercalés par des niveaux minces des grès qui sont déposés lors des écoulements faibles (diminution de vitesse) (Massari, 1983 ; Miall, 1985, 2006).

\section{Elément architectural OF "Overbank fines":-}

Presque toutes les coupes étudiées dans ce bassin contiennent cet élément architectural avec une variation d'épaisseur d'une région à l'autre. Il s'agit d'une association de lithofaciès fins à très fins (F5: Fl, F6: Fm et F4: Sh). L'élément architectural $\mathbf{O F}$ est considéré comme un élément majeur dans tout le bassin, son épaisseur peut dépasser la quarantaine de mètres. Il se présente sous forme de bancs épais ( 2 à 50 mètres d'épaisseur) de siltites et argilites violacées ou rouge-brique avec des niveaux de marmorisation. Ces siltites et argilites montrent parfois un aspect massif avec des laminations frustes indiquant un milieu de dépôt calme. Dans d'autres cas, elles montrent des laminations horizontales avec des intercalations très minces $(5 \mathrm{à} 30 \mathrm{~cm})$ de grès fins (faciès Overbank). D'après Miall (1985, 2006), dans la plupart des cas cet élément a une géométrie en forme de feuillet, ce qui reflète son origine par aggradation verticale. Au voisinage des chenaux actifs, ces feuillets sont séparés par des crevasses splays et présentent de faibles pendages de dépôts de levée. Le même auteur indique que les dépôts de cet élément peuvent également se déposer aux niveaux des chenaux abandonnés, à condition que sa limite basale soit concave et sa géométrie soit sous forme de lentille (Ethridge et al., 1981; Miall 1985 et 2006).

En plus de ces trois éléments architecturaux de Miall $(\mathbf{1 9 8 5}, \mathbf{1 9 9 6}, \mathbf{2 0 0 6})$ reconnus dans notre basin, deux autres associations de faciès non décrites par Miall, ont été caractérisés :

\section{Association de faciès de playa (AP):-}

Cette association de faciès (AP) a été identifiée dans la coupe de Kachlat Arian au voisinage du barrage de Oued El Maleh. C'est un assemblage de lithofaciès de 5 à $6 \mathrm{~m}$ d'épaisseur, il est formé par des alternances de siltites et argilites fines de couleur rouge brique (lithofaciès $\mathbf{F 5}: \mathbf{F l}$ et $\mathbf{F 6}: \mathbf{F m}$ ) et des grès fins montrant parfois des litages plans horizontaux (lithofaciès F4: Sh). L'association de faciès (AP) présente une cyclicité des faciès gréseux et silto-argileux ; ce qui montre que leur dépôt s'est fait au niveau de playa lake non salées. La présence des dépôts gréseux montre également que ces dernières étaient peu profonds (Liu et Wang, 2001).

\section{Association de faciès évaporitiques (AE):-}

Il s'agit d'une association de faciès argileux $(\mathbf{F 6}: \mathbf{F m})$ et évaporitiques (F7 : gypse) de 5 à $7 \mathrm{~m}$ d'épaisseur. Les épaisseurs de ces faciès varient entre 1 et 1,5 mètre pour les siltites et entre 10 à $20 \mathrm{~cm}$ pour le gypse. Ce faciès évaporitique a une origine souvent primaire et dans d'autres cas diagénétique. Il est formé par l'évaporation des eaux salines dans des vasières et des lagunes sous un climat chaud et aride en relation avec une mer pelliculaire en cours d'ouverture au Trias supérieur. 


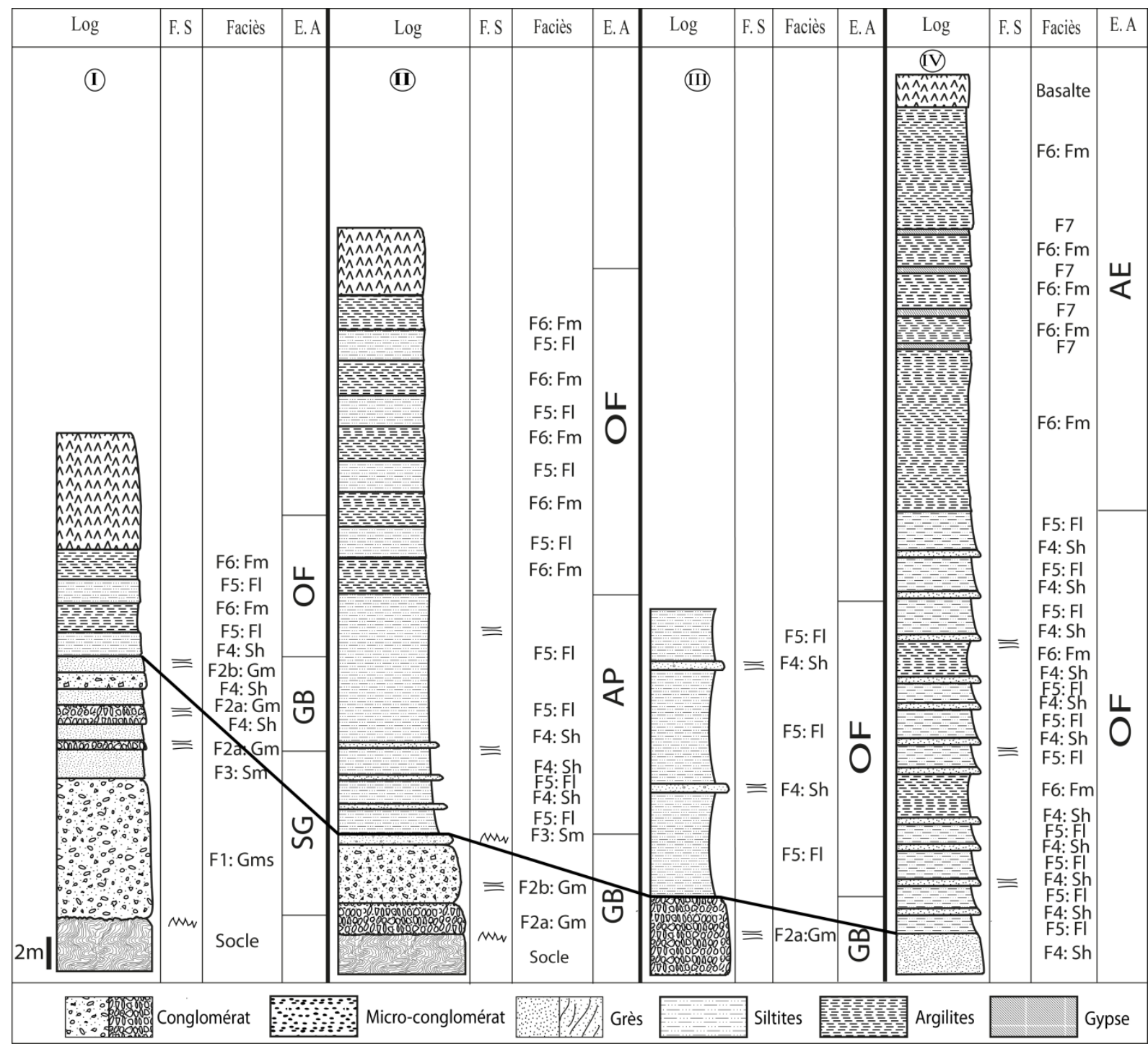

Figure 4:- Différentes coupes étudiées dans la région de l'Oued El Maleh-ElGara avec tous les lithofaciès (F) et les éléments architecturaux (E.A) caractérisés. I: coupe de Sidi Bouchaibe; II: coupe de Kachlat Arian; III: coupe de

Sidi Bou Amar; IV: coupe d'OuledJhaich dans la région d'ElGara.

\section{Environnements de depots:-}

L'analyse sédimentologique des dépôts triasiques dans la région d'Oued El Maleh et ElGara indique que les conditions fluviatiles ont nettement changé avec le temps. Dans la présente étude, des modèles conceptuels ont été construit pour donner une idée sur les environnements de dépôt des différents membres étudiés. Les structures sédimentaires internes, les surfaces de délimitation des lithofaciès et leurs associations verticales (éléments architecturaux) ont permis l'interprétation de ces environnements de dépôt.

Système en tresse proximal avec des cônes alluviaux: mieux représenté dans le secteur de Sidi Bouchaibe:-

Dans le secteurde Sidi Bouchaibe, les affleurements sont dominés essentiellement par des faciès grossiers et moyens à la base et au milieu: faciès F1: Gms, F2a: Gm, F2b: Gm, F3: Sm et F4: Sh. Deux principaux éléments architecturaux ont été formés par ces faciès, il s'agit des éléments architecturaux SG et GB. Le sommet de cette coupe est marqué par les faciès les plus fins ; ce sont généralement des siltites F5: Fl et des argilites F6: Fm plus ou moins stratifiées et formant l'élément architectural $\mathbf{O F}$. Donc, les dépôts grossiers de base ont été déposés au niveau de cônes alluviaux et de rivières graveleuses à écoulements gravitaires (modèle $\mathbf{N}^{\circ} \mathbf{1}$ de Miall, 1985, 2006). La présence des faciès silteux et argileux de l'élément $\mathbf{O F}$ au sommet de l'affleurement est probablement due à la 
migration des particules fines et leurs dépôt au niveau des chenaux abandonnés au voisinage des chenaux principaux.

Système en tresse avec des barres graveleuses: mieux représenté dansle secteur de Kachlat Arian:-

Cet affleurement est considéré parmi les plus puissants dans le bassin, son épaisseur maximale est de l'ordre de 80 à 100 mètres. Il est formé par des dépôts grossiers à la base (conglomérat stratifié à élément subanguleux et imbriqués localement montrant un dépôt de barre longitudinale), le faciès F2a: $\mathbf{~} \mathbf{m}$ est le premier faciès déposé sur le socle. Ensuite, il y a mise en place du faciès F2b: $\mathbf{G m}$ sur le quel est déposé le faciès Le F3: $\mathbf{S m}$. Cet ensemble de faciès formaient l'élément architectural GB. L'association de faciès AP (Association de Playa) est également identifiée. Il est formé dans ce secteur par des alternances de siltites fines et des grès très fins montrant un aspect des dépôts de playa. L'ensemble est surmonté par une alternance de faciès F5: Fl et F6: Fm sur une épaisseur de 60m donnant naissance à l'élément architectural $\mathbf{O F}$. D'après ces caractéristiques, les dépôts grossiers du base de la série ont été déposés dans un système fluviatile en tresse caractérisé par des barres graveleuses (modèle $\mathbf{N}^{\circ} \mathbf{2}$ de Miall (1985, 2006)). Les dépôts moyens et fins de l'élément architectural $\mathbf{O F}$ et de l'association des faciès $\mathbf{A P}$ caractérisés dans la partie médiane et sommitale de la série ont été probablement déposés dans une plaine d'inondation avec l'apparition de petits lacs.

Système anastomosé associé à une plaine côtière: mieux représenté dans le secteur d'ElGara:-

Dans ce secteur, l'affleurement d'Oulad Jhaich est formé par trois types de faciès de granulométrie fine à très fine, il s'agit de grès fins montrant parfois des litages plans horizontaux (lithofaciès F4: Sh), de siltites (lithofaciès F5: Fl), d'argilites (lithofaciès F6: Fm) qui sont les plus dominantes et d'évaporites (F7: gypse fibreux). Ces faciès sont associés en deux principaux éléments architecturaux qui sont $\mathbf{O F}$ quand il s'agit d'une association de faciès de grès, de siltites et d'argilites (Sh, Fl et $\mathbf{F m}$ ) et $\mathbf{A E}$ (Association de faciès évaporitique) quand il s'agit d'une association d'argilites et d'évaporites (gypse) avec parfois des passages très minces de grès très fin. Cette zone est caractérisée par la présence de dépôts de débordements formés au niveau des plaines d'inondations et des dépôts très fins et évaporitiques. Ce milieu de dépôt correspond probablement à la fin d'un système anastomosé avec une grande plaine côtière à l'aval caractérisée par le développement des playas lakes et des lagunes où sont formé les faciès évaporitiques (gypse) par l'évaporation des eaux marines sous un climat chaud et aride et en relation avec la Protoatlantique etla Téthys. 


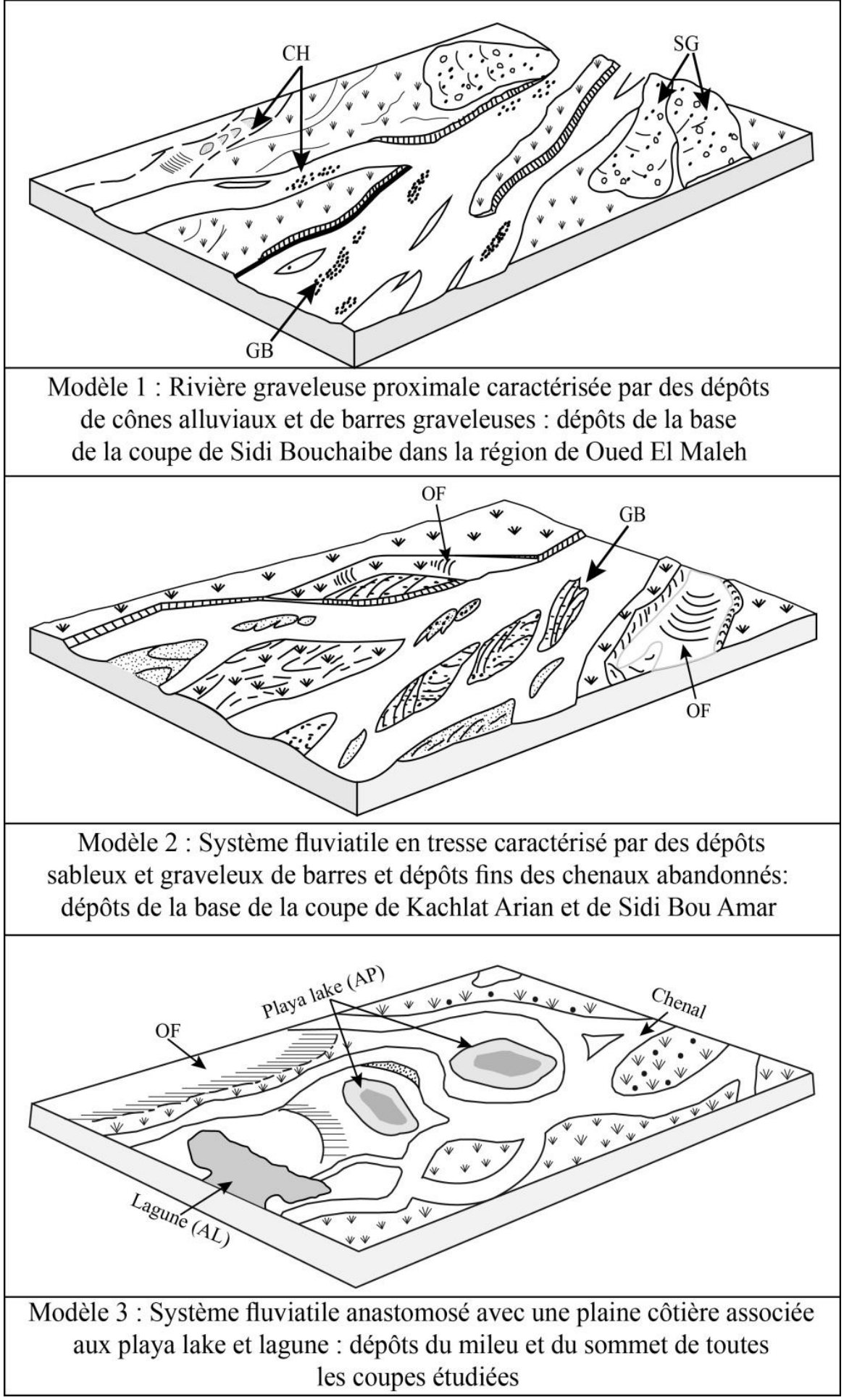

Figure 5:- Différents environnements de dépôts reconstitués dans les zones étudiées, le Modèle 1 et 2 sont inspirés de Miall (1985, 2006)

\section{Discussion:-}

Cette analyse sédimentologique basée sur les faciès, les éléments architecturaux, et les paléoenvironnements, montre que le bassin ouvert sous forme d'hemi-graben (El Wartiti and Fadli, 1985; El Wartiti et al., 1992; Lyazidi, 2004) est alimenté au début par des dépôts grossiers d'origine exclusivement continentale, déposés dans un système fluviatile proximal (cône alluviaux, système fluviatile en tresse). Ces dépôts détritiques proviennent de l'érosion des 
reliefs émergés de la Meseta et sont favorisés par des contrôles tectonique (failles bordières et failles synsédimentaires) et climatique sous régime fluviatile. Ensuite, une importante série argileuse (plus qu'une centaine de mètre d'épaisseur) a été mise en place. La puissante épaisseur de ces dépôts reflète leurs dépôts au niveau d'une plaine d'inondation dans un système anastomosé qui peut atteindre une plaine côtière.

Les niveaux du gypse observés en affleurement sont probablement déposés dans un système de plaine côtière et dans des milieux lagunaires. En profondeur, Peretsman et Holser (1988) ont parlé d'une épaisse série du sel, dont l'analyse du Brome montre leur originalité laguno-marine avec des influences continentales. La formation des dépôts fins et des évaporites est probablement liée à une élévation de niveau de base responsable du dépôt d'un système clastique rétrogradant au cours du Trias supérieur.

La sédimentation évaporitique a été stoppé brutalement (Trias supérieur-Lias inférieur) par l'épanchement des basaltes attribué à la CAMP "Central Atlantic magmatic province" et interprété comme étant lié au rifting de l'atlantique centrale, daté de 200 Ma dans ce bassin (Peretsman, 1985).

La comparaison entre la série triasique de notre région d'étude et les autres bassins de la marge atlantique (domaine mesetien) ainsi que celle dans le Haut Atlas marocain, a révélé plusieurs différences. La première différence concerne l'épaisseur de la série détritique, puisque au niveau du Haut Atlas la série est dominée par des formations conglomératiques et surtout gréseuses (Tixeront, 1973 ; Mattis, 1977 ; Biron, 1982 ; Beauchamp, 1982, 1988, 1995; Benaouiss et al., 1996; Hofmann et al., 2000), contrairement à la meseta qui est dominée par des dépôts silteux et argileux. La formation évaporitique dans les bassins mesetiens est également plus épaisse que dans le Haut Atlas, ceci est probablement due à l'importance et la proximité de cette mer pelliculaire fini-triasique qui est la proto-atlantique dans la meseta. La seconde différence se reflète en ce qui concerne l'origine des évaporites. Notre bassin ainsi que le bassin de Boufekrane et celui de Khémisset (par Et-Touhami, 1992 et 1998) montrent bien une origine commune. Les fortes teneurs en brome et les rapports isotopiques mesurés dans ces formation évaporitiques indiquent leur origine marine avec quelques influences continentales (Peretsman et Holser, 1988; Holser et al.,1988; Et-Touhami, 1992; Beauchamp et al., 1995). Par contre, le Haut Atlas est caractérisé par des évaporites continentales influencés par des apports marines mineures (Salvan, 1974 ; Biron, 1982). Concernant la série détritique basale, plusieurs auteurs ont parlé dans le Haut Atlas d'une série purement continentale (Van Houten, 1977 ; Manspeizer et al., 1978 ; Lorenz, 1988), malgré la présence de quelques fossiles d'eaux salées à saumâtres (Beauchamp, 1988). Dans notre zone d'étude, la formation gréso-conglomératique de base ne présente aucun indice marin jusqu'à ce jour.

\section{Conclusion:-}

Les dépôts triasiques de la région de Oued El Maleh-ElGara sont déposés dans un système fluviatile qui a connu une grande évolution dans le temps, il est passé d'un système proximal de cônes alluviaux marqué par des dépôts gravitaires «debris flow deposits» à un style fluviatile en tresse riche en dépôts de barres «Gravel Bar and Bedforms». Ce système était passé par la suite à un style anastomosé caractérisé par des sédiments moyens et fins de débordement. Ces environnements sont passés finalement à une plaine alluviale favorable pour l'accumulation des dépôts très fins d'overbank «Overbank fines», cette plaine alluviale associée à une plaine côtière où s'étaient développées probablement des playa lakes et des lagunes caractérisées par des dépôts très fins et des évaporites. Ces évaporites sont formées par l'évaporation des eaux salines sous un climat chaud et aride dans ce bassin considéré comme rift continental immergé par une mer pelliculaire au Trias supérieur.

\section{Références:-}

1. Allen, J.R.L., 1983. Studies in fluviatile sedimentation: bars, bar-complexes and sandstone sheets (lowsinuosity braided streams) in the Brownstones (L. Devonian), Welsh Borders. Sediment Geol, pp. 237-293.

2. Beauchamp, J., 1982. Le permien et le trias marocains : quelques acquisitions récentes. Département de Géologie, Faculté des Sciences de Marrakech

3. Beauchamp, J., 1988. Triassic sedimentation and rifting in the High Atlas (Morocco). In: Manspeizer W. (ed), Triassic-Jurassic Rifting: Continental Breakup and the Origin of the Atlantic Ocean and Passive Margins. Developments in Geotectonics, 22, p. 477-497.

4. Beauchamp, J., Benaouiss, N., \& Courel, L., 1995. Où était donc le domaine marin dans le Maroc africain au Trias supérieur. C. R. Acad. Sci. Paris, t. 221, sér. II a, p. 1033-1040. 
5. Benaouiss, N., Courel, L., Beauchamp, J., 1996. Rift-controlled fluvial/tidal transitional series in the Oukaimeden Sandstones, High Atlas of Marrakesh (Morocco).Sediment. Geol. 107, 21-36.

6. Biron, P.E., 1982. Le Permo-Trias de la région de l'Ourika (Haut-Atlas de Marrakech, Maroc):lithostratigraphie, sédimentologie, tectonique et minéralisations. Stratigraphy. UniversitéScientifiqueetMédicale de Grenoble. French. 〈tel-00710295〉

7. Bridge, J. S., 1993. Description and interpretation of fluvial deposits: a critical perspective. Sedimentology 40: 801-810.

8. Chalouan, A., 1981. Stratigraphie et structure du Paléozoïque de l'Oued Cherrat: un segment du couloir de cisaillement de Meseta occidentale (Maroc).Notes ServGéol Maroc 42 (308): 33-100.

9. Destombes, J., Jeannette, A., 1966. Mémoire explicatif de la carte géotechnique de la Meseta côtière à l'Est de Casablanca. Régions de Mohammedia, Bouznika et BenSlimane. Notes et Mémoires Service géologique Maroc, 180 bis, $104 p$.

10. Einsele, G., 2000. Sedimentary Basins: Evolution, Facies, and Sediment Budget. Springer, Berlin Heidelberg. $792 \mathrm{pp}$.

11. Eji Uba, C., Heubeck, C., Hulka C., 2005. Facies analysis and basin architecture of the Neogene Subandean synorogenic wedge, southern Bolivia. Sedimentary Geology.

12. El Wartiti, M., \& Fadli, D., 1985. Relations socle-couverture au cours du Trias dans la zone de BenSlimaneElGara (Maroc nord mesetien).Bull. Sci. Ter., Rabat, 1, 54-66.

13. El Wartiti, M., Medina, F., \&Fadli, D., 1992. Effects of the central Atlantic early rifting in the northern border of the Berrechid-ElGara basin (Morocco).Gaia, 4, 31-38.

14. Ethridge, F. G. et Flores, R. M., 1981. Recent and ancient nonmarine depositional environments: Models for exploration, Society of Economic Paleontologists and Mineralogists Special Publication 31, 349p.

15. Fadli D., 1990. Evolution sédimentaire et structurale des massifs du Khatouat et des Mdakra : Deux segments hercyniens de la Meseta nord-occidentale. Thèse de doctorat de Rabat Université Mohammed V, Rabat.

16. Hofmann, A., Tourani, A., Gaupp, R., 2000. Cyclicity of Triassic to Lower Jurassic continental red beds of the Argana Valley, Morocco; implications for palaeoclimate and basin evolution. Palaeogeogr. Palaeoclimatol. Palaeoecol. 161, 229-266.

17. Johnson, S.Y., 1984. Cyclic fluvial sedimentation in a rapidly subsiding basin, northwest Washington.Sediment Geol., 38: 361-392.

18. Liu, Z. et Wangb, C., 2001. Facies analysis and depositional systems of Cenozoic sediments in the Hoh Xil basin, northern Tibet. Sedimentary Geology 140, 251-270.

19. Lyazidi, A., 2004. Evolution géodynamique du bassin triasique de Berrechid-ElGara-BenSlimane (Meseta Nord Occidentale, Maroc). Thèse, Université Mohammed V-Agdal Rabat, Maroc.

20. Manspeizer, W., 1988. A stratigraphic record from Morocco and North America of rifting drifting and Tethyan transgressions of the Central Proto-Atlantic. Journal of African Earth Sciences, Vol. 7, No. 2, pp. 369-373.

21. Mattis, A.F., 1977. Nonmarine Triassic sedimentation, central High Atlas Mountains, Morocco. J. Sediment. Petrol. 47, 107-119.

22. Massari, F., 1983. Tabular cross-bedding in Messinian fluvial channel conglomerates, Southern Alps, Italy. In: J.D. Collinson and J. Lewin (Editors), Modern and Ancient Fluvial Systems. Int. Assoc. Sediment. Spec. Publ., 6: $287-300$.

23. Medina, F., 1996. Le Trias du Maroc: introduction. In Medina, F. (éditeur): le Permien et le Trias du Maroc. Editions PUMAG, Marrakech 1996, pp. 213-225.

24. Menkem, E.F., 2013. Lithofacies and depositional environment of volcano-sedimentary deposits of SE Mayo Oulo basin. American Journal of Geosciences 3 (2): 23-29.

25. Miall, A.D., 1977.A review of the braided river depositional environment. Earth-Sci. Rev., 13: 1-62.

26. Miall, A.D., 1978. Lithofacies types and vertical profile models in braided river deposits: a summary. In: A.D. Miall (Editor), Fluvial Sedimentology. Can. Soc. Petrol. Geol. Mem., 5: 597-604.

27. Miall, A.D., 1985. Architectural-Element Analysis: A New Method of Facies Analysis Applied to Fluvial Deposits. Earth-Science Reviews, 22 (1985) 261-308.

28. Miall, A.D., 2006. The Geology of Fluvial Deposits, Sedimentary Facies, Basin Analysis, and Petroleum Geology.4th corrected printing, Springer.

29. Opluštil, S., Martínek, K., Tasáryová, Z., 2005. Facies and architectural analysis of fluvial deposits of the Nýřany Member and the Týnec Formation (Westphalian D-Barruelian) in the Kladno-Rakovník and Pilsen basins. Bulletin of Geosciences, Vol. 80, $N^{\circ} 1,45-66$.

30. Piqué A., 1979. Evolution structurale d'un segment de la chaine hercynienne: la meseta marocaine nord occidentale. Sci. Géol., Mém.56. 
31. Poli, E., 1997. Stratigraphie séquentielle haute-résolution, modèles de dépôt et géométrie 2D-3D des séquences triasiques de la marge téthysienne ardéchoise. Thèse, Université de Bourgogne-Centre des Sciences de la Terre.

32. Postma, G., \& Cruickshank, C., 1988. Sedimentology of a late Weichselian to Holocene terraced fan delta, Varangerfjord, northern Norway. In Nemec, W. et Steel, R.J. (editors) Fan deltas: Sedimentology and tectonic settings. Blackie, London, 144-158.

33. Reineck, H. E. \& Singh, I. B., 1980. Depositional Sedimentary Environments. Springer-verlag, Berlin, Heidelberg, New York, $551 \mathrm{p}$.

34. Rust, B.R., 1972. Structure and Process in a braided river. Sedimentology, 18, 221-246.

35. Rust, B.R., 1978. Depositional models for braided alluvium. In: A.D. Miall (Editor), Fluvial Sedimentology. Can. Soc. Petrol. Geol. Mem., 5: 605-625.

36. Rust, B.R., 1981. Sedimentation in an arid-zone anastomosing fluvial system: Cooper's Creek, Central Australia. J. Sediment. Petrol.

37. Sohn, Y.K., Kim, S.B., Hwang, I.G., Bahk, J.J., Choe, M.Y., \& Chough, S.K., 1997. Characteristics and depositional processes of large-scale gravelly Gilbert-type foresets in the Miocene Doumsan fan delta, Pohang Basin, SE Korea. Journal of Sedimentary Research 67 (1), 130-141.

38. Sohn, Y.K., 2000. Depositional process of submarine debris flows in the Miocene fan deltas, Pohang Basin, SE Korea with special reference to flow transformation. Journal of Sedimentary Research, 70: 491-503.

39. Takahashi, T., 1978. Mechanical characteristics of debris flow. J. Hydraul. Div., Am. Soc. Civil Eng. 104, 1153-1169.

40. Takahashi, T., 1981. Debris flow. Ann. Rev. Fluid Mech. 13, 57-77.

41. Takahashi, T., 1991. Debris flows. IAHR Monograph Series. Balkema, Rotterdam.

42. Tixeront, M., 1973. Lithostratigraphie et minéralisation cuprifères et uranifères stratiformes syngenetiques et familières des formations détritiques permo-triasiques du Couloir d'Argana (Haut-Atlas occidental, Maroc). Notes Serv. géol. Maroc 249, 147-177.

43. Warren, J.K., 2006. Evaporites: Sediments, Resources and Hydrocarbons. Springer, New York, p. 1035.

44. Warren, J. K., 2010. Evaporites through time: Tectonic, climatic and eustatic controls in marine and nonmarine deposits. Earth-Science Reviews 98 pp. 217-268.

45. Zahraoui M., 1991. La plate-forme carbonatée dévonienne du Maroc occidental et sa dislocation-hercynienne. Thèse d'état, Université de Brest, $261 p$. 